\title{
Eating 'Junk-Food' Produces Rapid and Long-Lasting Increases in NAc CP-AMPA Receptors: Implications for Enhanced Cue-Induced Motivation and Food Addiction
}

\author{
Max F Oginsky', Paulette B Goforth', Cameron W Nobile', Luis F Lopez-Santiago' and Carrie R Ferrario*,I \\ 'Department of Pharmacology, University of Michigan Medical School, Ann Arbor, MI, USA
}

\begin{abstract}
Urges to eat are influenced by stimuli in the environment that are associated with food (food cues). Obese people are more sensitive to food cues, reporting stronger craving and consuming larger portions after food cue exposure. The nucleus accumbens (NAc) mediates cue-triggered motivational responses, and activations in the NAc triggered by food cues are stronger in people who are susceptible to obesity. This has led to the idea that alterations in NAc function similar to those underlying drug addiction may contribute to obesity, particularly in obesity-susceptible individuals. Motivational responses are mediated in part by NAc AMPA receptor (AMPAR) transmission, and recent work shows that cue-triggered motivation is enhanced in obesity-susceptible rats after 'junk-food' diet consumption. Therefore, here we determined whether NAc AMPAR expression and function is increased by 'junk-food' diet consumption in obesity-susceptible vs -resistant populations using both outbred and selectively bred models of susceptibility. In addition, cocaine-induced locomotor activity was used as a general 'read out' of mesolimbic function after 'junk-food' consumption. We found a sensitized locomotor response to cocaine in rats that gained weight on a 'junk-food' diet, consistent with greater responsivity of mesolimbic circuits in obesity-susceptible groups. In addition, eating 'junk-food' increased NAc calcium-permeable-AMPAR (CP-AMPAR) function only in obesity-susceptible rats. This increase occurred rapidly, persisted for weeks after 'junk-food' consumption ceased, and preceded the development of obesity. These data are considered in light of enhanced cue-triggered motivation and striatal function in obesity-susceptible rats and the role of NAc CP-AMPARs in enhanced motivation and addiction.
\end{abstract}

Neuropsychopharmacology (2016) 4I, 2977-2986; doi:I0.1038/npp.2016.1 II; published online 24 August 2016

\section{INTRODUCTION}

Although urges to eat are regulated by hunger, satiety, and energy demand, they are also strongly influenced by stimuli in the environment that are associated with food (food cues). For example, in non-obese people, exposure to food cues increases food craving and the amount of food consumed (Fedoroff et al, 1997; Soussignan et al, 2012). Obese people are more sensitive to these motivational properties of food cues, reporting stronger cue-triggered food craving and consuming larger portions after food cue exposure (Rogers and Hill, 1989; Yokum et al, 2011). These behavioral similarities between food- and drug-induced craving have led to the concept that 'food addiction' induced by the consumption of foods high in sugar and fat may contribute to the obesity epidemic (Carr et al, 2011; Epstein and Shaham, 2010; Kenny, 2011; Rogers and Hill, 1989; Volkow et al, 2013).

\footnotetext{
*Correspondence: Dr CR Ferrario, Department of Pharmacology, University of Michigan Medical School, A220B MSRB III, II 50 West Medical Center Drive, Ann Arbor, MI 48109-5632, USA, Tel: + 734 763 8637, Fax: + I 734763 4450, E-mail: Ferrario@umich.edu Received 9 February 2016; revised 14 June 2016; accepted 16 June 2016; accepted article preview online 7 July 2016
}

Evidence predominantly from human studies suggest that cue-triggered food craving in obese individuals involves alterations in function of the nucleus accumbens (NAc), a region that has long been known to mediate motivation for food and drug rewards, but that is increasingly implicated in obesity. For example, human fMRI studies show that activations in the NAc triggered by food cues are stronger in obese people (Stice et al, 2012; Volkow et al, 2013; Small, 2009). In addition, enhanced responsivity in the NAc to food cues predicts future weight gain and difficulty in losing weight in humans (Demos et al, 2012; Murdaugh et al, 2012). In rats, diet-induced obesity produces enhanced motivational responses to food cues, particularly in obesity-susceptible populations (Brown et al, 2015; Robinson et al, 2015). Together these data suggest that consumption of fatty, sugary foods produce neuroadaptations in NAc function that may enhance motivational processes.

In both rats and humans, susceptibility to obesity may have an important role in the effects of palatable, highcalorie 'junk-foods' on neural function and behavior (Albuquerque et al, 2015; Geiger et al, 2008; Robinson et al, 2015; Stice and Dagher, 2010). Although it is difficult to address the role of susceptibility in humans, studies in rats have shown that diet-induced alterations in mesolimbic systems and motivation are more pronounced in 
obesity-susceptible vs -resistant rats (Geiger et al, 2008; Vollbrecht et al, 2016; Robinson et al, 2015; Valenza et al, 2015; Oginsky et al, 2016). Thus recent data suggest that consumption of 'junk-foods' may produce distinct neural alterations in susceptible $v s$ resistant populations.

AMPA-type glutamate receptors (AMPARs) provide the main source of excitation to the NAc, and the ability of food cues to trigger food-seeking relies in part on activation of AMPARs in the NAc core (Di Ciano et al, 2001). Furthermore, consumption of sugary, fatty foods and obesity can alter excitatory transmission in the NAc (Tukey et al, 2013; Brown et al, 2015). In addition, recent work from our laboratory and others has shown that cue-triggered motivation is enhanced in obesity-susceptible populations (Robinson et al, 2015; Brown et al, 2015). The goal of the current study was to determine how junk-food consumption in obesity-susceptible and -resistant rats affects AMPAR expression and transmission in NAc core, as NAc AMPARs mediated cue-triggered drug-seeking but have not been examined in diet-induced obesity models. In addition, cocaine-induced locomotor activity was used as a general 'read out' of mesolimbic function, as enhanced responsivity of mesolimbic circuits increases the motivational impact of food cues (Wyvell and Berridge, 2000, 2001).

Two complementary rodent models were used in order to determine the role of susceptibility in 'junk-food'-induced alterations in NAc AMPARs. First, outbred Sprague-Dawley rats given 'junk-food' were identified as 'Gainers' and 'Non-Gainers' (as in Robinson et al, 2015), after which behavioral and neural differences were measured. Although informative, this model requires the induction of weight gain and diet manipulation in order to identify susceptible populations. Thus we also examined the effects of junk-food in rats selectively bred for their propensity or resistance to diet-induced obesity (Levin et al, 1997; Vollbrecht et al, 2015, 2016).

\section{MATERIALS AND METHODS}

\section{Subjects}

Rats were pair-housed on a reverse light-dark schedule $(12 / 12)$ with free access to food and water throughout and were aged 60-70 days at the start of the experiment. Male Sprague-Dawley rats were purchased from Harlan. Obesityprone and -resistant rats were bred in house. These lines were originally established by Levin et al (1997); breeders were purchased from Taconic. The inclusion of outbred rats enables comparisons to the broader existing literature, while selectively bred rats enable us to differentiate alterations owing to obesity $v s$ diet manipulation. Weight was measured 1-2 times per week. All procedures were approved by The UM Committee on the Use and Care of Animals.

\section{Junk-Food Diet and Identification of Obesity-Susceptible and -Resistant Outbred Rats}

The 'junk-food' is a mash of: Ruffles original potato chips (40 g), Chips Ahoy original chocolate chip cookies (130 g), Jif smooth peanut butter (130 g), Nesquik powdered chocolate flavoring (130 g), powdered Lab Diet 5001 (200 g; \% of calories: $19.6 \%$ fat, $14 \%$ protein, $58 \%$ carbohydrates; $4.5 \mathrm{kcal} / \mathrm{g}$ ), and

water $(180 \mathrm{ml})$ combined in a food processor. Diet composition is based on previous studies establishing subpopulations (Levin et al, 1997; Robinson et al, 2015). K-means clustering based on weight gain after 1 month of junk-food was used to identify obesity-susceptible (Junk-Food-Gainer) and obesityresistant (Junk-Food-Non-Gainer) groups. This statistical method provides an unbiased separation that can be applied uniformly across studies (MacQueen, 1967). In addition, we have determined that this is an optimal time point for reliably identifying subpopulations (Robinson et al, 2015; Oginsky et al, 2016; unpublished observations).

\section{Cocaine-Induced Locomotion}

Locomotor activity was measured in chambers $(41 \mathrm{~cm} \times 25.4 \mathrm{~cm} \times 20.3 \mathrm{~cm})$ equipped with photocell beams. Rats were placed in chambers for a $40 \mathrm{~min}$ habituation period prior to receiving an injection of saline $(1 \mathrm{ml} / \mathrm{kg}$, i.p.), followed $1 \mathrm{~h}$ later by cocaine $(15 \mathrm{mg} / \mathrm{kg}$, i.p.). This dose was chosen based on previous dose-response studies (Oginsky et al, 2016; Ferrario et al, 2005).

\section{Surface vs Intracellular Protein Expression}

Tissue from the NAc (core/shell) and dorsal medial striatum (DMS) were collected and processed using established $\mathrm{BS}^{3}$ crosslinking approaches (Boudreau et al, 2012) that enables the detection of cell surface $v s$ intracellular protein expression. DMS samples were included to determine whether differences were selective to the NAc. For each rat, tissue was isolated, chopped (McIllwain chopper; $400 \mu \mathrm{m}$ slices; St Louis, MO), and incubated in aCSF containing $2 \mathrm{mM}$ $\mathrm{BS}^{3}\left(30 \mathrm{~min}, 4^{\circ} \mathrm{C}\right)$. Crosslinking was terminated with glycine (100 mM; $10 \mathrm{~min})$, slices were homogenized in lysis buffer $(400 \mu \mathrm{l}$; in mM: $25 \mathrm{HEPES} ; 500 \mathrm{NaCl}, 2$ EDTA, 1 DTT, 1 phenylmethyl sulfonyl fluoride, $20 \mathrm{NaF}, 1: 100$ protease inhibitor cocktail set I (Calbiochem, San Diego, CA), and $0.1 \%$ Nonidet P-40 [v/v]; $\mathrm{pH} 7.4)$, and stored at $-80^{\circ} \mathrm{C}$. Protein concentration was determined by BCA assay. See Boudreau et al (2012) for full methodological details.

$\mathrm{BS}^{3}$ crosslinked samples were heated in Laemmli sample treatment buffer with $5 \% \beta$-mercaptoethanol $\left(70{ }^{\circ} \mathrm{C}, 10 \mathrm{~min}\right)$, loaded $(20 \mu \mathrm{g}$ protein), and electrophoresed on $4-15 \%$ BisTris gradient gels under reducing conditions. Proteins were transferred onto PVDF membranes (Amersham Biosciences, Piscataway, NJ). Membranes were rinsed, blocked (1 h, RT, $5 \%(\mathrm{w} / \mathrm{v})$ with nonfat dry milk in TBS-Tween 20 (TBS-T; $0.05 \%$ Tween $20, \mathrm{v} / \mathrm{v})$ ), and incubated overnight $\left(4^{\circ} \mathrm{C}\right)$ with primary antibodies (1:1000 in TBS) to GluA1 (Thermo Scientific; PA1-37776) or GluA2 (NeuroMab, UCDavis/NIH: 75-002). Membranes were washed in TBS-T, incubated with HRP-conjugated secondary (Invitrogen, Carlsbad, CA; $1 \mathrm{~h}$, $\mathrm{RT}$ ), washed, and immersed in chemiluminescence-detecting substrate (GE Healthcare, Piscataway, NJ). Images were acquired on film, and Ponceau S (Sigma-Aldrich) was used to determine total protein. Bands of interest were quantified using Image $\mathrm{J}(\mathrm{NIH})$.

\section{Electrophysiology}

The $\mathrm{BS}^{3}$ crosslinking procedure described above provides information about surface expression (synaptic and extra 
synaptic) of individual AMPAR subunits, whereas electrophysiological data provide information about functional synaptic AMPARs (tetramers). Whole-cell patch-clamp recordings of medium spiny neurons (MSNs) in the NAc core were conducted after junk-food exposure in outbred and selectively bred rats. Prior to slice preparation, rats were anesthetized with chloral hydrate $(400 \mathrm{mg} / \mathrm{kg}$, i.p.), brains were rapidly removed and placed in ice-cold oxygenated $\left(95 \% \mathrm{O}_{2}-5 \% \mathrm{CO}_{2}\right.$ ) aCSF containing (in $\left.\mathrm{mM}\right): 125 \mathrm{NaCl}, 25$ $\mathrm{NaHCO}_{3}, 12.5$ glucose, $1.25 \mathrm{NaH}_{2} \mathrm{PO}_{4}, 3.5 \mathrm{KCl}, 1 \mathrm{~L}$-ascorbic acid, $0.5 \mathrm{CaCl}_{2}, 3 \mathrm{MgCl}_{2}$, and $305 \mathrm{mOsm}, \mathrm{pH}$ 7.4. Coronal slices $(300 \mu \mathrm{m})$ containing the NAc were made using a vibratory microtome (Leica Biosystems, Buffalo Grove, IL, USA) and allowed to rest in oxygenated aCSF (40 min). For the recording aCSF $(2 \mathrm{ml} / \mathrm{min}), \mathrm{CaCl}_{2}$ was increased to $2.5 \mathrm{mM}$ and $\mathrm{MgCl}_{2}$ was decreased to $1 \mathrm{mM}$. Patch pipettes were pulled from $1.5 \mathrm{~mm}$ borosilicate glass capillaries (WPI, Sarasota, FL; 3-7 M $\Omega$ resistance) and filled with a solution containing (in mM): $140 \mathrm{CsCl}, 10$ HEPES, $2 \mathrm{MgCl}_{2}, 5 \mathrm{Na}^{+}$ATP, $0.6 \mathrm{Na}^{+}$-GTP, 2 QX314, pH 7.3, and $285 \mathrm{mOsm}$. Recordings were conducted in the presence of picrotoxin $(50 \mu \mathrm{M})$. Evoked EPSCs (eEPSCs) were elicited by local stimulation $(0.05-0.30 \mathrm{~mA}$ square pulses, $0.3 \mathrm{~ms}$, delivered every $20 \mathrm{~s}$ ) using a bipolar electrode placed $\sim 300 \mu \mathrm{m}$ lateral to recorded neurons. The minimum amount of current needed to elicit a synaptic response with $<15 \%$ variability in amplitude was used. If $>0.30 \mathrm{~mA}$ was required, the neuron was discarded. AMPAR-mediated eEPSCs were recorded at $-70 \mathrm{mV}$ before and after application of the CP-AMPAR selective antagonist naspm $(200 \mu \mathrm{M}$; as in Conrad et al, 2008; Ferrario et al, 2011).

\section{Statistics}

Two-tailed $t$-tests, one-way or two-way repeated-measures ANOVAs, Sidak's post-hoc multiple comparisons tests, and planned comparisons between obesity-susceptible and -resistant groups were used (Prism 6, GraphPad, San Diego, CA).

\section{RESULTS}

\section{Experiment 1}

Sprague Dawley rats were given junk-food using an approach that leads to obesity in some rats (Junk-Food Gainers) but not others (Junk-Food Non-Gainers; Robinson et al, 2015; Oginsky et al, 2016). We then measured the response to a single cocaine injection (a general readout of mesolimbic function), surface $v s$ intracellular expression of AMPAR subunits, and AMPAR-mediated transmission in the NAc core using whole-cell patch clamping approaches in these two populations.

Greater cocaine-induced locomotion in Junk-FoodGainers. As expected, when given junk-food some rats gained a substantial amount of weight (Junk-Food-Gainers, $N=6$ ) while others did not (Junk-Food-Non-Gainers, $N=4$; Figure 1a; two-way RM ANOVA: main effect of group: $\mathrm{F}_{(1,9)}=11.85, p=0.007$; group $\times$ time interaction: $\left.\mathrm{F}_{(18,162)}=6.85, p<0.001\right)$. These rats had access to junkfood for 5 months total to allow for maximal separation between groups. They were then returned to standard laboratory chow (Lab Diet 5001: $4 \mathrm{kcal} / \mathrm{g} ; 4.5 \%$ fat, $23 \%$ protein, $48.7 \%$ carbohydrates; percentage of caloric content) for a 2 week junk-food deprivation period to evaluate differences that persist after junk-food removal. Next rats were given a single cocaine injection and locomotor activity was monitored; the purpose of this was to obtain a general readout of mesolimbic function. The response to cocaine was greater in Junk-Food-Gainers vs Junk-Food-Non-Gainers (Figure 1b; two-way RM ANOVA: group $\times$ time interaction: $\mathrm{F}_{(21,168)}=2.31, p=0.0018$; Sidak's test, $\left.{ }^{*} p<0.05\right)$. In addition, while Junk-Food-Gainers showed a significantly stronger locomotor response to cocaine than saline (two-way RM ANOVA, time $\times$ injection interaction: $\left.\mathrm{F}_{(6,30)}=2.39, p<0.05\right)$, Junk-Food-Non-Gainers did not. Locomotion during habituation and after saline did not differ between groups (Figure 1b inset), consistent with previous reports (Oginsky et al, 2016; Robinson et al, 2015).

GluA1, but not GluA2, NAc surface expression is greater in Junk-Food-Gainers. Next, we examined surface and intracellular protein expression of AMPAR subunits in JunkFood-Gainers and Junk-Food-Non-Gainers. The majority of AMPARs in the NAc are GluA1/GluA2 containing, with some GluA2/3 AMPARs, and a small number of GluA2lacking, CP-AMPARs ( 10\%; Reimers et al, 2011; Scheyer et al, 2014). So we focused on GluA1 and GluA2 expression levels, as this provides a good indication of changes in these different AMPAR populations. The abundance of surface and intracellular GluA1 and GluA2 protein was measured 1 week after testing for cocaine-induced locomotor activity (Figure 1c-e). Previous studies have established that a single cocaine injection does not alter AMPARs at this time (Boudreau and Wolf, 2005; Ferrario et al, 2010; Kourrich et al, 2007), enabling us to interpret AMPAR differences as related to the diet (see also below). NAc surface expression of GluA1 was greater in Junk-Food-Gainers vs Junk-Food-NonGainers (Figure $1 \mathrm{~d} ; t_{8}=2.7, p=0.03$ ). In contrast, NAc GluA2 expression did not differ between groups (Figure 1e). In addition, GluA1 and GluA2 expression in the DMS of these same rats was similar between groups (data not shown), suggesting that changes in AMPAR expression occur selectively in the NAc. An increase in NAc GluA1 surface expression in the absence of changes in surface GluA2 suggests the presence of CP-AMPARs (GluA1/1- or GluA1/3-containing receptors). However, this must be confirmed using electrophysiological methods. We therefore conducted whole-cell patch clamp recordings after junk-food exposure to determine whether there is an increase in the contribution of CP-AMPARs to synaptic transmission in the NAc of Junk-Food-Gainers.

$C P$-AMPAR-mediated transmission is increased in JunkFood-Gainers. For electrophysiological experiments, a separate cohort of rats was given junk-food for 3 months and recordings were made after 3 weeks of junk-food deprivation. This procedure was chosen to minimize overcrowding in cages due to weight gain, and to examine relatively long-lasting effects of junk-food. In this cohort, all junk-food rats were 'Gainers', gaining even more weight than Junk-Food-Gainers within cohort 1 (3-month gain: cohort 1, 106.2 $\pm 9.7 \mathrm{~g}$; cohort 2, $\sim 132 \pm 5.4 \mathrm{~g}$ ). Therefore, 
a

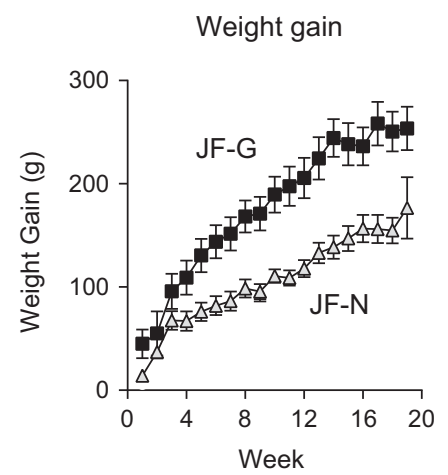

b Greater cocaine-induced locomotion in JF-Gainers

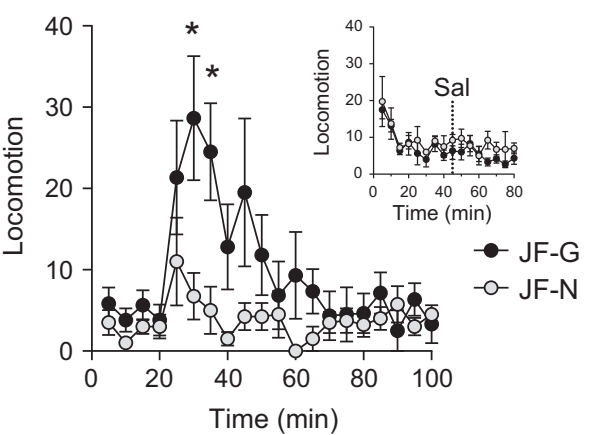

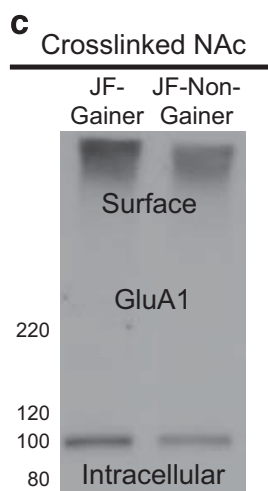

d

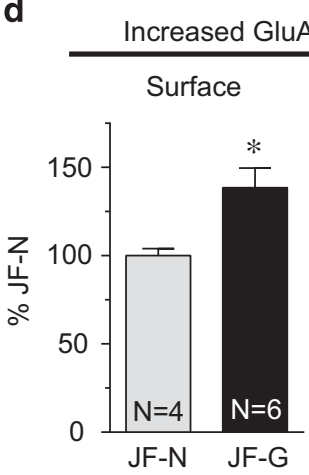

e $\frac{\text { No change in GluA2 expression }}{\text { Surface }}$

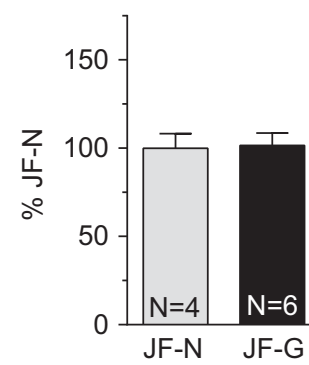

Figure I GluAI, but not GluA2, surface expression is greater in Junk-Food-Gainers than Non-Gainers. (a) Junk-food produces substantial weight gain in a subset of susceptible rats. (b) Eating junk-food followed by junk-food deprivation is associated with a sensitized response to cocaine in Junk-Food-Gainers (JFG) compared with Junk-Food-Non-Gainers (JF-N). Inset shows locomotion during habituation and after saline injection. (c) Representative blot of GluAI expression in crosslinked NAc samples. (d, e) GluAI, but not GluA2, surface expression is greater in Junk-Food-Gainers compared with Junk-Food-NonGainers after junk-food deprivation, suggesting the presence of CP-AMPARs. All data are shown as mean \pm SEM; $* p<0.05$.
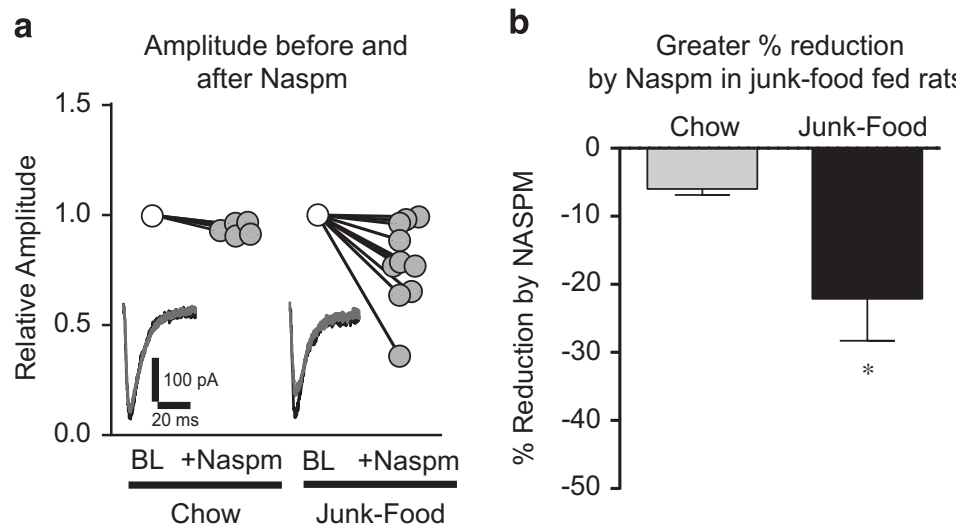

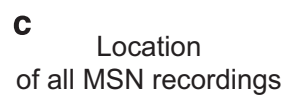
of all MSN recordings

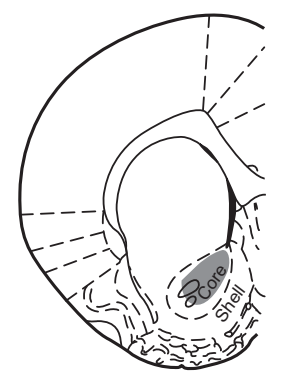

Figure 2 The contribution of CP-AMPARs is greater in Junk-Food-Gainer vs chow-fed rats following junk-food deprivation. (a) Normalized amplitude before (BL) and after bath application of the CP-AMPAR antagonist naspm $(200 \mu \mathrm{M})$. Inset shows example eEPSCs before (black) and after naspm (red). (b) The reduction by naspm is greater in Junk-Food-Gainer vs chow-fed rats. (c) Location of whole-cell recordings for all experiments. The shaded area indicates the general location of recordings made in the NAc core. Recordings fell approximately between 2.04 and 1.56 mm from Bregma; figure adapted from Paxinos and Watson (2007). All data shown as mean \pm SEM; $* p<0.05$. A full color version of this figure is available at the Neuropsychopharmacology journal online.

comparisons were made between the Chow ( $N=5$ cells, 3 rats $)$ and Junk-Food-Gainer groups $(N=10$ cells, 7 rats $)$. To assess the contribution of CP-AMPARs to total AMPARmediated synaptic transmission, we used the selective CP-AMPAR antagonist naspm $(200 \mu \mathrm{M})$. Naspm produced a small reduction in eEPSC amplitude in the Chow-fed controls (Figure 2a; Two-way ANOVA: main effect of naspm, $\left.\mathrm{F}_{(1,13)}=19.14, p=0.0008\right)$, consistent with prior reports that CP-AMPARs contribute 5-10\% of the basal AMPAR-mediated eEPSC (eg, Scheyer et al, 2014). However, in the junk-food 

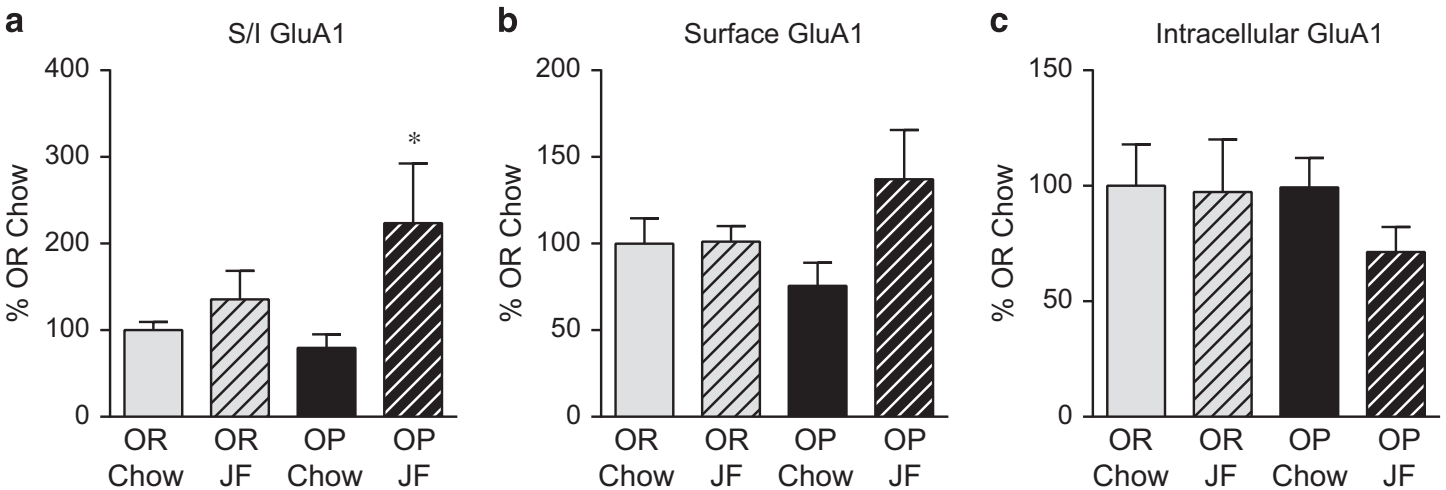

Figure 3 The relative abundance of NAc GluAI surface vs intracellular (S/I) protein expression is enhanced after junk-food consumption and deprivation only in obesity-prone rats. This was due to shifts in both surface and intracellular protein expression. (a) Surface to intracellular ratio, (b) surface and (c) intracellular expression of GluAI protein in obesity-resistant $(\mathrm{OR})$ and obesity-prone (OP) rats given chow or junk-food. All data shown as mean \pm SEM; * $p<0.05$ : OP-JF vs OP-Chow.

group, naspm produced a significantly greater reduction (Figure $2 \mathrm{~b} ; t_{13}=1.8 ; p=0.046$ ). These data show that $\mathrm{CP}$ AMPARs are increased in Junk-Food-Gainers compared with Chow-fed rats. Furthermore, as the cohort used for electrophysiology was not given cocaine, these data strongly suggest that the biochemical changes in the previous experiment reflected effects of junk-food, not the single cocaine exposure.

\section{Experiment 2}

Data above from outbred rats are consistent with the idea that junk-food preferentially increases CP-AMPARs in obesity-susceptible rats. However, this difference could be due to the development of obesity or to preexisting differences in susceptible rats. To address these possibilities, we conducted similar biochemical and electrophysiological studies in selectively bred obesity-prone and -resistant rats with and without junk-food exposure. Because we know $a$ priori which rats are susceptible to obesity, we can use this model to differentiate preexisting differences $v s$ changes induced by junk-food.

Basal GluA1 levels are similar, but junk-food increases GluA1 expression in obesity-prone rats. First, we examined NAc AMPAR expression in obesity-prone and -resistant rats given chow or junk-food. NAc tissue was collected and crosslinked after 1 month of junk-food followed by 1 month of junk-food deprivation. A shorter junk-food exposure was used here to increase feasibility of experiments, as selectively bred obesity-prone rats tend to gain weight more rapidly than the outbred population. GluAl expression was similar in obesity-prone and -resistant rats given chow (Figure 3, solid bars; $N=6$ /group), suggesting that baseline levels of GluA1-containing AMPARs are similar in susceptible rats. This is consistent with previous electrophysiological results showing that basal AMPAR-mediated transmission is similar in these rats (Oginsky et al, 2016). In the junk-food fed groups, the abundance of surface to intracellular (S/I) GluA1 expression was increased in obesity-prone, but not obesityresistant, rats compared with chow-fed controls (Figure 3a: one-way ANOVA, $\mathrm{F}_{(3,19)}=2.957, p=0.058$; OP-Chow $v s$ OP-JF, $p<0.05$; OP-JF $N=5$, OR-JF $N=6$ ). This increase in S/I was due to slight increases in GluA1 surface expression
(Figure 3b) and slight reductions in intracellular GluA1 (Figure 3c). Again, no differences were found in GluA2 expression (data not shown). Results here are consistent with biochemical results above in outbred rats and show that differences in AMPAR expression in obesity-prone rats are the result of junk-food and not due to basal differences between obesity-prone and -resistant groups.

Junk-food increases NAc CP-AMPAR-mediated transmission in obesity-prone rats in the absence of differences in weight or junk-food consumption. Next we determined whether junkfood consumption in the absence of weight gain is sufficient to enhance NAc AMPARs. A separate cohort of selectively bred rats were given chow or junk-food for 9-10 days (to minimize the development of obesity) followed by 2 weeks of junk-food deprivation and measurement of CP-AMPAR-mediated transmission as described above. Naspm reduced the AMPARmediated eEPSC amplitude in all groups (Figure 4a; Two-way RM ANOVA: main effect of naspm: $\mathrm{F}_{(1,20)}=22.5, p=0.0001$; group $\times$ drug interaction: $\mathrm{F}_{(3,20)}=4.29, p=0.02$; OP-JF and OR-JF: $N=7$ cells, 5 rats; OP-Chow: $N=4$ cells, 3 rats; ORChow $N=5$ cells, 3 rats). However, the effect of naspm was significantly greater in obesity-prone rats given junk-food compared with all other groups (Figure 4b: two-way RM ANOVA, group $\times$ time interaction: $\mathrm{F}_{(18,114)}=2.87, p=0.0003$; ${ }^{*} p<0.05$ OP-JF $v s$ all other groups; Figure 4c: one-way ANOVA, $\mathrm{F}_{(3,20)}=9.53, p=0.0004$; OP-JF $v s$ OR-JF and OPChow $v s$ OP-JF, $p<0.01)$. In addition, the effect of naspm was similar in the OP-Chow, OR-Chow, and OR-JF groups and was comparable to that seen in outbred rats (above) and to previously reported basal CP-AMPAR transmission (Conrad et al, 2008; Scheyer et al, 2014). Furthermore, weight gain, weight on recording day, and the amount of junk-food consumed was similar between obesity-prone and -resistant groups (Figure $4 \mathrm{~d}$ and e). Thus, these data show that consumption of junk-food preferentially increases CPAMPARs in obesity-prone rats prior to the onset of differential weight gain.

One possibility is that junk-food produces CP-AMPAR upregulation in obesity-resistant rats but that this effect subsides after 2 weeks of junk-food deprivation. To address this, recordings were made after 1 day of junk-food 
a Naspm sensitive AMPAR-
mediated current

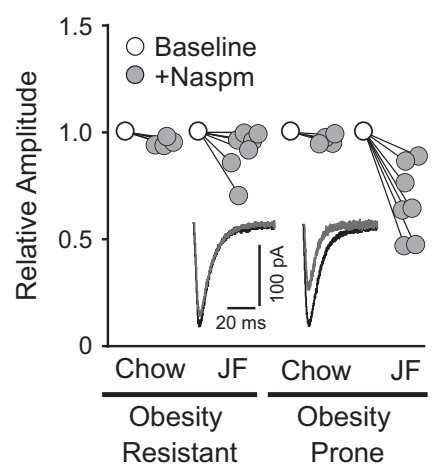

d

Weight gain is similar between groups

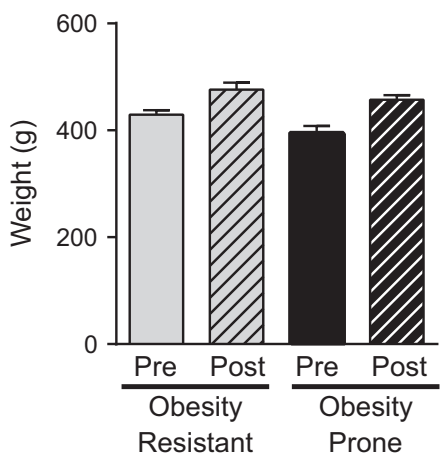

Time course before and after $\quad \mathbf{C}$ bath application of NASPM
JF increases CP-AMPAR

mediated transmission in

obesity-prone rats
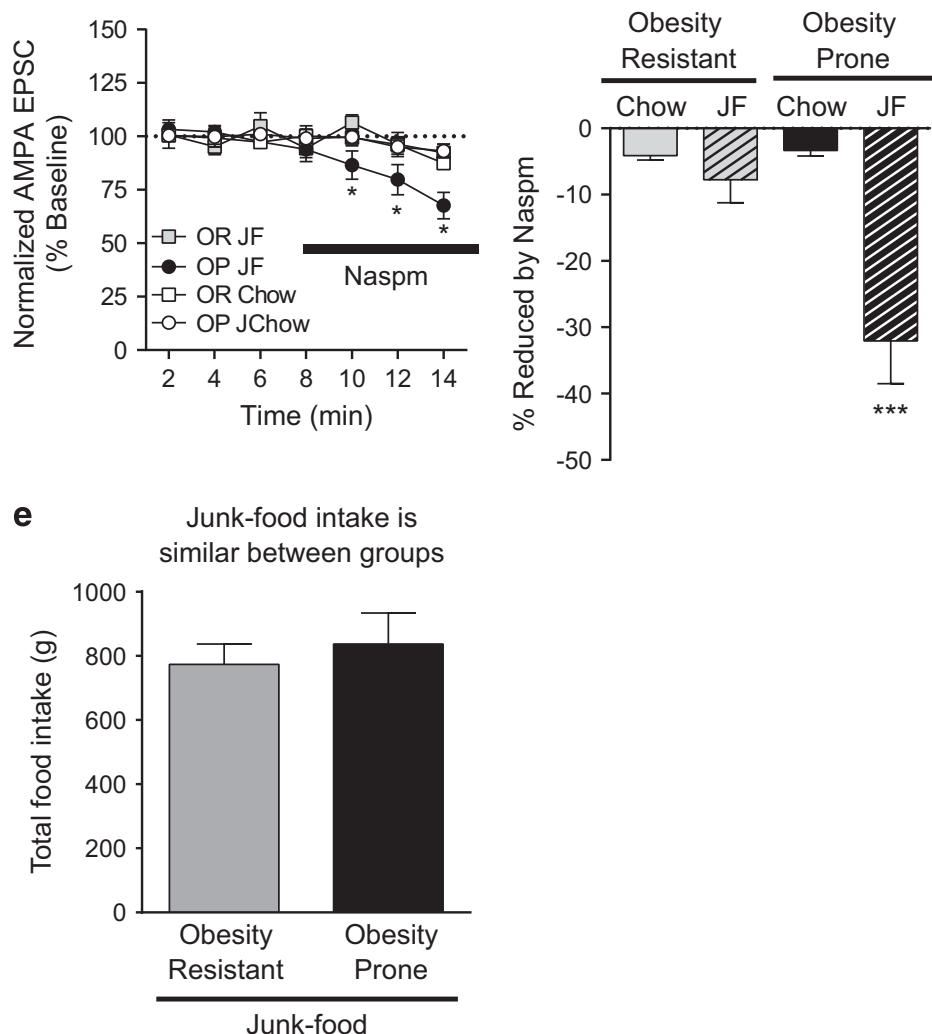

Figure 4 Just 10 days of junk-food followed by 2 weeks of junk-food deprivation is sufficient to induce CP-AMPAR upregulation in obesity-prone but not obesity-resistant rats. This increase occurred in the absence of differences in food intake and weight gain. (a) Normalized amplitude before and after naspm $(200 \mu M)$. Inset: Example of eEPSCs from junk-food fed rats before (black) and after naspm (red). (b) Time course of eEPSC before and after naspm application. (c) The reduction by naspm is increased after junk-food in obesity-prone but not obesity-resistant rats. (d) Weight gain is similar between groups. (e) Junk-food consumption is similar between groups. All data shown as mean \pm SEM. $* p<0.05$; $* * * * 0<0.00$ I OP-JF vs all other groups. A full color version of this figure is available at the Neuropsychopharmacology journal online.

deprivation in another cohort of obesity-prone and -resistant rats given the same junk-food exposure (9-10 days; OR-JF: $N=7$ cells, 4 rats; OP-JF: $N=6$ cells, 3 rats). Again, we found that the effect of naspm was much greater in the OP-JF group (Figure 5a; two-way RM ANOVA: main effect of naspm: $\mathrm{F}_{(1,11)}=53.94, p<0.0001$; group $\times$ naspm interaction: $\mathrm{F}_{(1,11)}=13.75, p=0.0035$; Figure 5b: main effect of naspm: $\mathrm{F}_{(7,77)}=13.39, \quad p<0.0001 ; \quad$ group $\times$ naspm interaction: $\mathrm{F}_{(7,77)}=7.57, p<0.0001$, post-test ${ }^{*} p<0.05$; Figure $5 \mathrm{c}$ : unpaired $t$-test: $p=0.001)$. In addition, the magnitude of naspm's effect in the OR-JF group was comparable to chow controls. Together these data show that junk-food induced increases in CP-AMPARs are absent in obesity-resistant rats after both early and late deprivation periods. Furthermore, weight gain and food intake were again similar in obesityprone and -resistant rats (Figure $5 \mathrm{~d}$ and e). Thus junk-food induced increases in CP-AMPARs in obesity-prone rats are not due to weight gain or differences in the amount of junk-food consumed. Finally, no differences were found in baseline eEPSC amplitude across all the groups studied (Figure $5 \mathrm{f}$ one-way ANOVA baseline amplitudes: $\left.\mathrm{F}_{(7,44)}=1.993, p=0.09\right)$. Thus differences in naspm sensitivity above are not due to differences in baseline responding.
Raw amplitudes before and after naspm for all data are shown in Figure 5f.

\section{DISCUSSION}

Enhanced cue-triggered urges to eat and changes in mesolimbic function are thought to contribute to human obesity. Here we found that general responsivity of mesolimbic circuits is enhanced in rats that are susceptible to diet-induced obesity. In addition, junk-food increased NAc CP-AMPAR function in obesity-susceptible rats. This increase was present after 1, 14, or 21 days of junk-food deprivation, suggesting that CPAMPAR upregulation occurs rapidly and persists long-after junk-food consumption ceases. Further, the duration of junkfood exposure did not correspond to the magnitude of CPAMPAR increases in obesity-susceptible rats. Finally, this upregulation occurred more readily in obesity-susceptible rats and preceded the development of obesity.

\section{Greater Responsivity of Mesolimbic Systems in Obesity-Susceptible Rats}

After junk-food deprivation, cocaine-induced locomotion was greater in Junk-Food-Gainers than Non-Gainers, ie, Junk-Food- 

AMPAR-mediated current

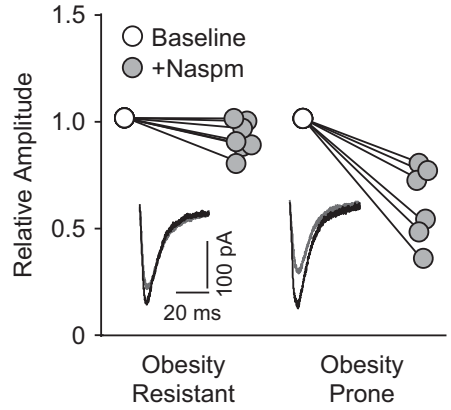

d

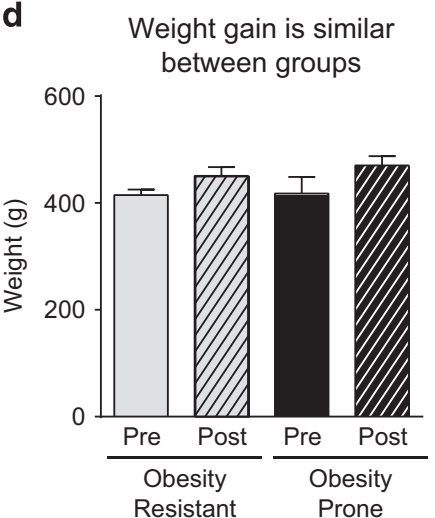

b

Time course before and after bath application of Naspm

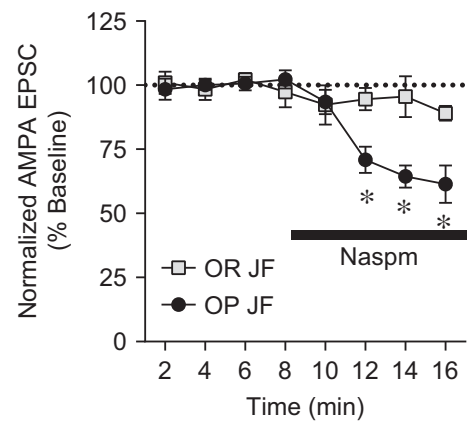

e

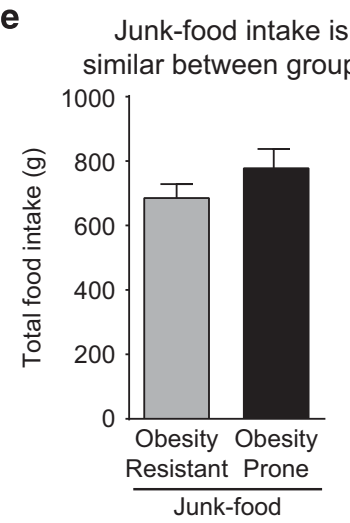

C CP-AMPAR transmission is increased after 1 day of JF-deprivation

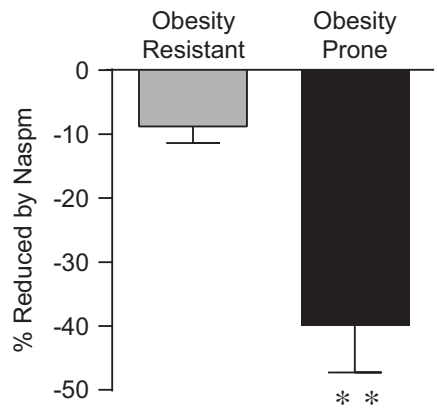

Summary of individual amplitudes across all studies

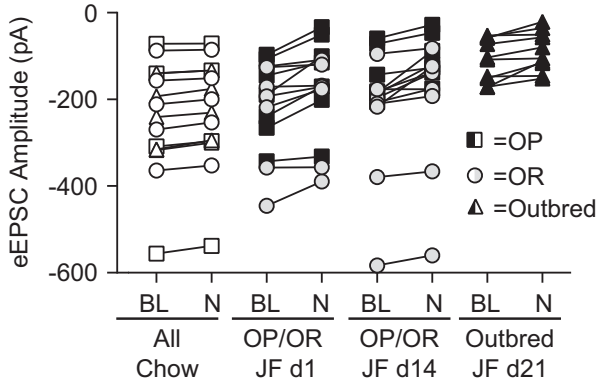

Figure 5 Junk-food-induced increases in CP-AMPARs are present after just I day of junk-food deprivation in obesity-prone but not obesity-resistant rats. (a) Normalized amplitude before (Baseline) and after naspm (200 $\mu$ M). Inset: Example eEPSCs from junk-food fed rats before (black) and after naspm (red). (b) Time course before and after naspm application. (c) The reduction by naspm is greater in obesity-prone vs obesity-resistant rats given junk-food. (d) Weight gain is similar between groups. (e) Junk-food consumption is similar between groups. All data are shown as mean $\pm \mathrm{SEM}$. * $=p<0.05$, ** $p<0.0 \mathrm{I}$. ( $\mathrm{f}$ ) Summary of individual eEPSC amplitudes across all studies (BL=baseline, $N=+$ naspm; open symbols =chow groups, closed symbols $=$ junk-food groups, triangles =outbred rats, circles=obesity-resistant rats, and squares=obesity-prone rats). A full color version of this figure is available at the Neuropsychopharmacology journal online.

Gainers were sensitized compared with Non-Gainers. Locomotor sensitization is indicative of alterations in the function of mesolimbic circuits that enhance incentive motivation for food and drug rewards (Robinson and Berridge, 2008; Vezina, 2004; Wolf and Ferrario, 2010). Thus the sensitized response found here is consistent with enhanced mesolimbic function and increased motivational responses previously reported in obesitysusceptible rats (Robinson et al, 2015; Brown et al, 2015). Importantly, differences in cocaine-induced locomotion are not likely due to differences in the levels of cocaine achieved. Specifically, using the same dose as in the current study, we have shown that the concentration of cocaine in the striatum is similar between obesity-prone and -resistant rats regardless of weight differences (Vollbrecht et al, 2016) and that obese $v s$ non-obese outbred rats that differ substantially in weight show the same locomotor response to cocaine prior to junk-food deprivation (Oginsky et al, 2016).

Sensitization in Junk-Food-Gainers may be due to differing effects of junk-food on mesolimbic systems in obesitysusceptible rats or may reflect preexisting differences. Consistent with preexisting differences, selectively bred obesity-prone rats are more sensitive to the locomotor-activating effects of cocaine than obesity-resistant rats prior to any diet manipulation (Oginsky et al, 2016; Vollbrecht et al, 2016). In addition, when tested after junk-food exposure but without junk-food deprivation, amphetamine- and cocaine-induced locomotion are similar between Junk-Food-Gainers and Junk-Food-Non-Gainers but enhanced compared with chow-fed controls (Oginsky et al, 2016; Robinson et al, 2015). Together, these data suggest that mesolimbic systems are sensitized in obesity-susceptible rats prior to diet manipulation and that junk-food consumption induces neuroadaptations that may further enhance reactivity in mesolimbic systems (see Oginsky et al, 2016; Vollbrecht et al, 2016 for further discussion).

\section{Junk-Food Selectively Increases NAc CP-AMPAR- Mediated Transmission in Obesity-Prone Rats}

When differences in surface $v s$ intracellular expression of NAc AMPAR subunits were examined, we found increases in GluA1, but not GluA2, surface expression in obesitysusceptible rats. This pattern was found in outbred rats identified as Junk-Food-Gainers and in selectively bred obesity-prone rats given free access to junk-food. Importantly, biochemical and electrophysiological data from controls show that basal levels of AMPAR expression and function are similar in selectively bred obesity-prone and -resistant groups, consistent with previous 
electrophysiological data (Oginsky et al, 2016). Thus differences in AMPAR subunit expression are likely due to the diet manipulation and not to basal differences between obesity-susceptible and -resistant groups (see also below).

As mentioned above, the majority of NAc AMPARs are GluA1/GluA2 or GluA2/GluA3 containing, with GluA2lacking CP-AMPARs comprising only 10\% of AMPARs (Reimers et al, 2011; Scheyer et al, 2014; see also Wolf and Tseng, 2012 for a review). Thus, an increase in GluA1 surface expression without changes in GluA2 expression after junk-food consumption in susceptible rats suggested a dietinduced increase in CP-AMPARs. To directly measure CP-AMPAR-mediated transmission, we used whole-cell patch clamping approaches in NAc core and measured differences in sensitivity to the selective CP-AMPAR antagonist, naspm, in the junk-food and chow-fed groups. We found that junk-food consumption increased sensitivity to naspm in obesity-susceptible, but not obesity-resistant, rats. Specifically, CP-AMPARs contributed to $\sim 10 \%$ of the current in Junk-Food-Non-Gainers and in chow-fed obesityprone and -resistant rats, consistent with previous reports, but was significantly upregulated in Junk-Food-Gainers and obesity-prone rats exposed to junk-food. Interestingly, a similar magnitude of CP-AMPAR upregulation was found regardless of the duration of exposure ( 3 months, 1 month, or 10 days). Furthermore, this increase was present after 1, 14 , or 21 days of junk-food deprivation, suggesting that CP-AMPAR upregulation occurs rapidly and persists longafter junk-food consumption ceases.

We next determined whether weight gain or eating junkfood itself was responsible for this long-lasting increase in CP-AMPARs. This experiment requires the use of selectively bred rats, as diet-induced weight gain is used to identify susceptible outbred rats. Obesity-prone and -resistant rats were given junk-food for just 9-10 days before recordings were made. This produced similar weight gain and junkfood intake in both groups. However, CP-AMPAR-mediated transmission was still significantly increased only in obesityprone rats. Thus junk-food more readily increased CPAMPAR-mediated transmission in obesity-prone rats. In addition, the fact that this increase precedes the development of obesity suggests that this neural change may drive subsequent behavioral differences (see also below). Of course, this does not preclude the possibility that additional plasticity may accompany the development of obesity.

Although few studies have examined the role of susceptibility, one study using an 'incubation' of cue-induced sucrose 'craving' model found a reduction in the NAc AMPA/NMDA ratio 21 days after the last sucrose selfadministration session (Counotte et al, 2014). In contrast, a separate study showed that sucrose consumption produced immediate (within $24 \mathrm{~h}$ ) but modest increases in CPAMPARs in the NAc (Tukey et al, 2013). Although several procedural differences likely contribute, one noteworthy difference is that Counotte et al (2014) used sagittal sections in which PFC inputs to the NAc were predominantly stimulated, whereas the current study and that of Tukey et al (2013) used coronal slices in which a mix of glutamatergic inputs were stimulated. This raises the interesting possibility that CP-AMPAR upregulation may be restricted to distinct glutamatergic inputs to the NAc (see also Lee et al, 2013; Ma et al, 2014). This should be addressed in future studies.
The mechanism(s) that induce long-lasting increases in NAc CP-AMPARs are poorly understood. However, we recently found that intrinsic excitability of MSNs in NAc core is enhanced in obesity-prone $v s$-resistant rats (Oginsky et al, 2016). This may lower the threshold for plasticity induction in obesity-prone individuals. For example, activation of D1-dopamine receptors enhances AMPAR surface expression (Wolf et al, 2003) and palatable foods increase NAc dopamine levels. Thus junk-food-induced elevations in dopamine may contribute to CP-AMPAR upregulation, although it is still unclear what governs a selective longterm enhancement of CP- vs non-CP-AMPARs.

To our knowledge, no studies have examined alterations in AMPARs in the NAc shell after diet manipulations comparable to those used here. However, one study has found that a high-fat diet does not alter dendritic spine density in the NAc shell (Dingess et al, 2016). The core and shell have differing roles in food-seeking $v s$ eating and receive distinct glutamatergic inputs (Sesack and Grace, 2010). Thus the possibility that effects may differ in these subregions should be investigated in future.

\section{What is the Functional Significance of CP-AMPAR Upregulation?}

In addition to affecting subsequent plasticity (Cull-Candy et al, 2006), AMPARs mediate cue-triggered food-seeking behaviors (Di Ciano et $a l, 2001$ ) and CP-AMPARs in the NAc core mediate enhanced cue-triggered cocaine-seeking in the incubation of 'craving' model (Wolf and Tseng, 2012; Wolf, 2016). We recently found that obesity-susceptible rats show enhanced approach, greater invigoration of foodseeking (PIT) and greater conditioned reinforcement in response to a food cue after junk-food consumption (Robinson et al, 2015; and unpublished observations). These behaviors are mediated in part by glutamatergic transmission in the NAc. Thus we speculate that increases in NAc CPAMPARs induced by consumption of sugary, fatty foods may contribute to enhanced cue-triggered food-seeking in obesity-susceptible populations. Of course, this hypothesis needs to be directly tested, but it is consistent with the role of CP-AMPARs in cue-triggered cocaine-seeking.

There are some noteworthy differences between food- and cocaine-induced upregulation of CP-AMPARs. Cocaineinduced increases in NAc core CP-AMPARs require prolonged exposure to intravenous cocaine and at least 3 weeks of withdrawal (Wolf and Tseng, 2012). In contrast, the increase found here occurred after just 1 day of junk-food deprivation and only 9-10 days of junk-food exposure. The ability of junk-food to produce immediate and long-lasting changes in CP-AMPARs is somewhat surprising given that repeated i.p. cocaine or amphetamine or limited access to cocaine self-administration do not increase CP-AMPARs (Nelson et al, 2009; Wolf and Tseng, 2012). Furthermore, the magnitude of junk-food-induced increases in CP-AMPARs is comparable to increases found after prolonged cocaine self-administration and withdrawal that mediate enhanced cue-triggered cocaine-seeking ( $40 \%$ here and $\sim 30 \%$ after cocaine withdrawal). Although direct comparisons to cocaine are difficult to make, it appears that junk-food may more readily induce CP-AMPAR upregulation than cocaine and/or may produce this increase via different mechanisms. 
Is AMPAR Upregulation Related to Enhanced Cocaine-Induced Locomotion in Obesity-Susceptible Rats?

Although greater cocaine-induced locomotion in obesitysusceptible rats is consistent with enhanced mesolimbic function, it is unlikely that this is due to changes in AMPAR expression or function. First, sensitivity to cocaine-induced locomotion is enhanced in selectively bred obesity-prone rats when AMPAR expression and function do not differ between these groups (Oginsky et al, 2016; Vollbrecht et al, 2016; current results). In addition, previous studies have shown that locomotor sensitization induced by repeated cocaine injection produces increases in AMPAR expression and function but that this change does not directly mediate the expression of locomotor sensitization (Ferrario et al, 2010). Rather, experience-induced increases in NAc AMPAR expression and function are more closely related to enhanced incentive motivation (Wang et al, 2013; Ferrario et al, 2010; Wolf and Ferrario, 2010).

\section{Summary and Future Directions}

We show that eating junk-food more readily increases NAc CP-AMPAR expression and function in obesity-susceptible rats. We speculate that CP-AMPAR upregulation contributes to previously observed increases in cue-triggered motivation in obesity-susceptible and obese populations (eg, Robinson et al, 2015), although direct tests of this should be conducted in future. Given the ongoing discussion about the contribution of 'food addiction' to obesity (Brown et al, 2015; Carr et al, 2011; Epstein and Shaham, 2010; Kenny, 2011; Volkow et al, 2013), it will be important to determine to what extent these food-induced changes in striatal function may be part of normal, adaptive processes $v s$ maladaptive, 'addictive-like' behaviors.

\section{FUNDING AND DISCLOSURE}

Cocaine was provided by the NIDA drug supply program. This work was supported by NIDDK R01DK106188 to CRF; MFO was supported by NIDA T32DA007268. Research support to PBG was provided by the Michigan Diabetes Research Center (NIH Grant P30 DK020572) and the Michigan Nutrition and Obesity Research Center (P30 DK089503). The authors declare no conflict of interest.

\section{REFERENCES}

Albuquerque D, Stice E, Rodriguez-Lopez R, Manco L, Nobrega C (2015). Current review of genetics of human obesity: from molecular mechanisms to an evolutionary perspective. Mol Genet Genomics 290: 1190-1221.

Boudreau AC, Milovanovic M, Conrad KL, Nelson C, Ferrario CR, Wolf ME (2012). A protein cross-linking assay for measuring cell surface expression of glutamate receptor subunits in the rodent brain after in vivo treatments. Curr Protoc Neurosci Chapter 5: Unit 5.30.1-5.30.19.

Boudreau AC, Wolf ME (2005). Behavioral sensitization to cocaine is associated with increased AMPA receptor surface expression in the nucleus accumbens. J Neurosci 25: 9144-9151.

Brown RM, Kupchik YM, Spencer S, Garcia-Keller C, Spanswick DC, Lawrence AJ et al (2015). Addiction-like synaptic impairments in diet-induced obesity. Biol Psychiatry (e-pub ahead of print).
Carr KA, Daniel TO, Lin H, Epstein LH (2011). Reinforcement pathology and obesity. Curr Drug Abuse Rev 4: 190-196.

Conrad KL, Tseng KY, Uejima JL, Reimers JM, Heng LJ, Shaham Y et al (2008). Formation of accumbens GluR2-lacking AMPA receptors mediates incubation of cocaine craving. Nature 454: $118-121$.

Counotte DS, Schiefer C, Shaham Y, O'Donnell P (2014). Time-dependent decreases in nucleus accumbens AMPA/NMDA ratio and incubation of sucrose craving in adolescent and adult rats. Psychopharmacology 231: 1675-1684.

Cull-Candy S, Kelly L, Farrant M (2006). Regulation of Ca2 +-permeable AMPA receptors: synaptic plasticity and beyond. Curr Opin Neurobiol 16: 288-297.

Demos KE, Heatherton TF, Kelley WM (2012). Individual differences in nucleus accumbens activity to food and sexual images predict weight gain and sexual behavior. J Neurosci 32: 5549-5552.

Di Ciano P, Cardinal RN, Cowell RA, Little SJ, Everitt BJ (2001). Differential involvement of NMDA, AMPA/kainate, and dopamine receptors in the nucleus accumbens core in the acquisition and performance of pavlovian approach behavior. J Neurosci 21: 9471-9477.

Dingess PM, Darling RA, Kurt Dolence E, Culver BW, Brown TE (2016). Exposure to a diet high in fat attenuates dendritic spine density in the medial prefrontal cortex. Brain Struct Funct (e-pub ahead of print).

Epstein DH, Shaham Y (2010). Cheesecake-eating rats and the question of food addiction. Nat Neurosci 13: 529-531.

Fedoroff IC, Polivy J, Herman CP (1997). The effect of pre-exposure to food cues on the eating behavior of restrained and unrestrained eaters. Appetite 28: 33-47.

Ferrario CR, Gorny G, Crombag HS, Li Y, Kolb B, Robinson TE (2005). Neural and behavioral plasticity associated with the transition from controlled to escalated cocaine use. Biol Psychiatry 58: 751-759.

Ferrario CR, Li X, Wang X, Reimers JM, Uejima JL, Wolf ME (2010). The role of glutamate receptor redistribution in locomotor sensitization to cocaine. Neuropsychopharmacology 35: 818-833.

Ferrario CR, Loweth JA, Milovanovic M, Ford KA, Galinanes GL, Heng LJ et al (2011). Alterations in AMPA receptor subunits and TARPs in the rat nucleus accumbens related to the formation of $\mathrm{Ca}(2)(+)$-permeable AMPA receptors during the incubation of cocaine craving. Neuropharmacology 61: 1141-1151.

Geiger BM, Behr GG, Frank LE, Caldera-Siu AD, Beinfeld MC, Kokkotou EG et al (2008). Evidence for defective mesolimbic dopamine exocytosis in obesity-prone rats. FASEB J 22: 2740-2746.

Kenny PJ (2011). Common cellular and molecular mechanisms in obesity and drug addiction. Nat Rev Neurosci 12: 638-651.

Kourrich S, Rothwell PE, Klug JR, Thomas MJ (2007). Cocaine experience controls bidirectional synaptic plasticity in the nucleus accumbens. J Neurosci 27: 7921-7928.

Lee BR, Ma YY, Huang YH, Wang X, Otaka M, Ishikawa M et al (2013). Maturation of silent synapses in amygdala-accumbens projection contributes to incubation of cocaine craving. Nat Neurosci 16: 1644-1651.

Levin BE, Dunn-Meynell AA, Balkan B, Keesey RE (1997). Selective breeding for diet-induced obesity and resistance in SpragueDawley rats. Am J Physiol 273(2 Pt 2): R725-R730.

Ma YY, Lee BR, Wang X, Guo C, Liu L, Cui R et al (2014). Bidirectional modulation of incubation of cocaine craving by silent synapse-based remodeling of prefrontal cortex to accumbens projections. Neuron 83: 1453-1467.

MacQueen JB. Some Methods for classification and Analysis of Multivariate Observations. Proceedings of 5th Berkeley Symposium on Mathematical Statistics and Probability. University of California Press: Berkeley, CA, 1966, pp 281-297. 
Murdaugh DL, Cox JE, Cook EW 3rd, Weller RE (2012). fMRI reactivity to high-calorie food pictures predicts short- and long-term outcome in a weight-loss program. Neuroimage 59: 2709-2721.

Nelson CL, Milovanovic M, Wetter JB, Ford KA, Wolf ME (2009). Behavioral sensitization to amphetamine is not accompanied by changes in glutamate receptor surface expression in the rat nucleus accumbens. J Neurochem 109: 35-51.

Oginsky MF, Maust JD, Corthell JT, Ferrario CR (2016). Enhanced cocaine-induced locomotor sensitization and intrinsic excitability of NAc medium spiny neurons in adult but not in adolescent rats susceptible to diet-induced obesity. Psychopharmacology 233: 773-784.

Paxinos G, Watson CJ. The Rat Brain in Stereotaxic Coordinates, 6th edn. Academic Press: Burlington, MA, USA, 2007.

Reimers JM, Milovanovic M, Wolf ME (2011). Quantitative analysis of AMPA receptor subunit composition in addiction-related brain regions. Brain Res 1367: 223-233.

Robinson MJ, Burghardt PR, Patterson CM, Nobile CW, Akil H, Watson SJ et al (2015). Individual differences in cue-induced motivation and striatal systems in rats susceptible to diet-induced obesity. Neuropsychopharmacology 40: 2113-2123.

Robinson TE, Berridge KC (2008). Review. The incentive sensitization theory of addiction: some current issues. Philos Trans $R$ Soc Lond Ser B Biol Sci 363: 3137-3146.

Rogers PJ, Hill AJ (1989). Breakdown of dietary restraint following mere exposure to food stimuli: interrelationships between restraint, hunger, salivation, and food intake. Addict Behav 14: 387-397.

Scheyer AF, Wolf ME, Tseng KY (2014). A protein synthesisdependent mechanism sustains calcium-permeable AMPA receptor transmission in nucleus accumbens synapses during withdrawal from cocaine self-administration. J Neurosci 34: 3095-3100.

Sesack SR, Grace AA (2010). Cortico-basal ganglia reward network: microcircuitry. Neuropsychopharmacology 35: 27-47.

Small DM (2009). Individual differences in the neurophysiology of reward and the obesity epidemic. Int J Obesity 33: S44-S48.

Soussignan R, Schaal B, Boulanger V, Gaillet M, Jiang T (2012). Orofacial reactivity to the sight and smell of food stimuli. Evidence for anticipatory liking related to food reward cues in overweight children. Appetite 58: 508-516.

Stice E, Dagher A (2010). Genetic variation in dopaminergic reward in humans. Forum Nutr 63: 176-185.

Stice E, Figlewicz DP, Gosnell BA, Levine AS, Pratt WE (2012). The contribution of brain reward circuits to the obesity epidemic. Neurosci Biobehav Rev 37(Pt A): 2047-2058.

Tukey DS, Ferreira JM, Antoine SO, D'Amour JA, Ninan I, Cabeza de Vaca $S$ et al (2013). Sucrose ingestion induces rapid AMPA receptor trafficking. J Neurosci 33: 6123-6132.

Valenza M, Steardo L, Cottone P, Sabino V (2015). Diet-induced obesity and diet-resistant rats: differences in the rewarding and anorectic effects of D-amphetamine. Psychopharmacology 232: $3215-3226$.
Vezina P (2004). Sensitization of midbrain dopamine neuron reactivity and the self-administration of psychomotor stimulant drugs. Neurosci Biobehav Rev 27: 827-839.

Volkow ND, Wang GJ, Tomasi D, Baler RD (2013). Obesity and addiction: neurobiological overlaps. Obes Rev 14: 2-18.

Vollbrecht PJ, Mabrouk OS, Nelson AD, Kennedy RT, Ferrario CR (2016). Pre-existing differences and diet-induced alterations in striatal dopamine systems of obesity-prone rats. Obesity 24: 670-677.

Vollbrecht PJ, Nobile CW, Chadderdon AM, Jutkiewicz EM, Ferrario CR (2015). Pre-existing differences in motivation for food and sensitivity to cocaine-induced locomotion in obesityprone rats. Physiol Behav 152(Pt A): 151-160.

Wang X, Cahill ME, Werner CT, Christoffel DJ, Golden SA, Xie Z et al (2013). Kalirin-7 mediates cocaine-induced AMPA receptor and spine plasticity, enabling incentive sensitization. J Neurosci 33: 11012-11022.

Wolf ME (2016). Synaptic mechanisms underlying persistent cocaine craving. Nat Rev Neurosci 17: 351-365.

Wolf ME, Ferrario CR (2010). AMPA receptor plasticity in the nucleus accumbens after repeated exposure to cocaine. Neurosci Biobehav Rev 35: 185-211.

Wolf ME, Mangiavacchi S, Sun X (2003). Mechanisms by which dopamine receptors may influence synaptic plasticity. Ann NY Acad Sci 1003: 241-249.

Wolf ME, Tseng KY (2012). Calcium-permeable AMPA receptors in the VTA and nucleus accumbens after cocaine exposure: when, how, and why? Front Mol Neurosci 5: 72.

Wyvell CL, Berridge KC (2000). Intra-accumbens amphetamine increases the conditioned incentive salience of sucrose reward: enhancement of reward 'wanting' without enhanced 'liking' or response reinforcement. J Neurosci 20: 8122-8130.

Wyvell CL, Berridge KC (2001). Incentive sensitization by previous amphetamine exposure: increased cue-triggered 'wanting' for sucrose reward. J Neurosci 21: 7831-7840.

Yokum S, Ng J, Stice E (2011). Attentional bias to food images associated with elevated weight and future weight gain: an fMRI study. Obesity (Silver Spring) 19: 1775-1783.

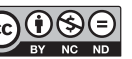

This work is licensed under a Creative Commons Attribution-NonCommercial-NoDerivs $\quad 4.0$ International License. The images or other third party material in this article are included in the article's Creative Commons license, unless indicated otherwise in the credit line; if the material is not included under the Creative Commons license, users will need to obtain permission from the license holder to reproduce the material. To view a copy of this license, visit http://creativecommons.org/licenses/by-nc-nd/4.0/

(C) The Author(s) 2016 